\title{
Dynamics of matter-wave solitons in a ratchet potential
}

\author{
Dario Poletti, ${ }^{1,2}$ Tristram Alexander, ${ }^{2}$ Elena Ostrovskaya, ${ }^{2}$ Baowen Li,,${ }^{1,3}$ and Yuri S. Kivshar ${ }^{2}$ \\ ${ }^{1}$ Department of Physics and Centre for Computational Science and Engineering, \\ National University of Singapore, Singapore 117542, Republic of Singapore \\ ${ }^{2} A R C$ Centre of Excellence for Quantum-Atom Optics and Nonlinear Physics Centre, \\ The Australian National University, Canberra ACT 0200, Australia \\ ${ }^{3}$ NUS Graduate School for Integrative Sciences and Engineering, 117597, Republic of Singapore
}

(Dated: October 26, 2018)

\begin{abstract}
We study the dynamics of bright solitons formed in a Bose-Einstein condensate with attractive atomic interactions perturbed by a weak bichromatic optical lattice potential. The lattice depth is a biperiodic function of time with a zero mean, which realises a flashing ratchet for matter-wave solitons. The average velocity of a soliton and the directed soliton current induced by the ratchet depend on the number of atoms in the soliton. We employ this feature to study collisions between ratchet-driven solitons and find that soliton transport can be induced through their interactions. In the regime when matter-wave solitons are narrow compared to the lattice period the ratchet dynamics is well described by the effective Hamiltonian theory.
\end{abstract}

PACS numbers: 03.75.Lm, 03.75.Kk, 05.60.-k

The ratchet effect, i.e. rectified average current induced by an asymmetric potential and unbiased zeromean driving, has historically attracted a lot of attention due to its possible relevance to biological transport, and molecular motors, and prospects for nanotechnology [1, 2]. Both in classical and quantum systems, the ratchet effect has been studied both in dissipative and Hamiltonian regimes [3, 4] and it appears to be due to a broken space-time symmetry of the perturbing potential [5]. Directed transport can be experimentally implemented in a range of different physical systems ranging from semiconductor hetero-structures to quantum dots, Josephson junctions, and cold atoms in optical lattices [6]. Recently, the interest in optical ratchets and especially in the effect of interaction on the ratchet transport [7, 8, 9] has resurged with the experimental advances in implementing atomic ratchets for Bose-Einstein condensates [10, 11].

As a physical system with intrinsically present nonlinear interactions, Bose-Einstein condensates (BECs) of atoms with negative scattering length support the existence of localized collective excitations - bright matterwave solitons. It is therefore interesting to explore the effect of the ratchet potentials on the transport and interaction properties of such collective excitations. It is especially important because theoretical studies of the soliton ratchets so far were mostly focused on topological solitons [12], whereas BECs represent a perfect test-bed for the study of the ratchet dynamics of a general class of non-topological solitons described by a continuous GrossPitaevskii (or nonlinear Schrödinger) equation.

In this Letter, we study, for the first time to our knowledge, the effect of the ratchet potential on nontopological non-dissipative bright matter-wave solitons. A BEC represents an intrinsically nonlinear system which displays a single-particle behavior on a macroscopic scale being easily manipulated by reconfigurable optical and magnetic potentials. The ratchet potential is realized by means of a bichromatic optical lattice which is "flashed" on and off in such a way that its time-averaged amplitude vanishes. We show that both the ratchet effect and soliton directed transport are observed in this system even in the absence of losses, which sets it apart from previously studied dissipative nonlinear systems subjected to ratchet potentials [13]. A weak potential does not affect the soliton shape in a wide range of parameters, and especially in the regime when the solitons are strongly localized, which justifies treatment of the soliton as an effective classical particle. However, we show that the ratchet still works even when the extended nature of the excitation cannot be ignored and the soliton width exceeds the period of the lattice. Moreover, we investigate the influence of the ratchet on soliton scattering, and show that multiple collisions between solitons may provide a space averaging mechanism that can enable observation of a soliton current in a ratchet potential.

To study the dynamics of bright solitons in attractive BECs with ratchet potentials we consider a soliton formed in a strongly elongated condensate cloud [14] subjected to a flashing one-dimensional optical lattice. As long as the energy of the longitudinal excitations is not sufficient to excite the transverse modes of the condensate, the system can be treated as one-dimensional and described by the Gross-Pitaevskii (GP) equation:

$$
i \frac{\partial \Psi}{\partial t}+\frac{1}{2} \frac{\partial^{2} \Psi}{\partial x^{2}}+|\Psi|^{2} \Psi-V(x, t) \Psi=0,
$$

where the Fourier-synthesized lattice potential,

$$
V(x, t)=V_{0} f(t)[\cos (x)+\cos (2 x+\phi)],
$$

is driven biperiodically: $f(t)=\sin (\omega t)+\sin (2 \omega t)$, and $V_{0}$ depends on the intensity of the laser beams forming 
the lattice. Quantum transport of atoms in the stationary potential of the form (2) has been recently studied experimentally with ultracold rubidium vapor [15]. With the time-dependent potential (2) the space inversion symmetry is broken for $\phi \neq 0, \pi$ and the time inversion symmetry is broken by our choice of $f(t)$. According to the symmetry analysis [5], this can allow a directed transport of matter-wave solitons.

In derivation of the dimensionless model (1) we have assumed that the energy, length, and frequency are measured in the units of $E_{0}=\hbar^{2} k^{2} / m, a_{0}=1 / k$, and $\omega_{0}=\hbar k^{2} / m$, respectively, where $m$ is the mass of the atoms, and $k$ is the wavevector of the optical lattice. We have furthermore reduced the three-dimensional meanfield model to the one-dimensional GP equation by assuming that the wavefunction is separable in the following manner: $\psi_{3 D}(x, y, z)=\psi_{1 D}(x) \Phi(y, z)$, where $\Phi(y, z)$ is the ground state wavefunction, normalized to one, of the two-dimensional harmonic oscillator with the transverse trapping frequency $\omega_{\perp}$. With these assumptions, the wavefunction $\Psi$ in Eq. (11) relates to $\psi_{1 D}$ as follows: $\Psi=\psi_{1 D} \sqrt{g_{1 D}}$, where $g_{1 D}=2\left(a_{s} \omega_{\perp}\right) /\left(a_{0} \omega_{0}\right)$ is the renormalized interaction coefficient that characterizes s-wave scattering of the condensate atoms with the scattering length $a_{s}$. Number of atoms in the system is defined as: $\mathcal{N}=N / g_{1 D}$, where $N=\int|\Psi|^{2} d x$ is the norm of the dimensionless one-dimensional wavefunction.

In the experimental setup [14] a bright soliton forms in the ${ }^{7} \mathrm{Li}$ cloud with a modified scattering length $a_{s} \approx-0.21 \mathrm{~nm}$ trapped in a quasi-one-dimensional atomic waveguide with $\omega_{\perp}=2 \pi \times 710 \mathrm{~Hz}$. We consider this experimental setup with an additional optical lattice formed by light beams crossed at the angle $\theta=38^{\circ}$, derived from a $\mathrm{CO}_{2}$ laser with the wavelength $\lambda=10.62 \mu \mathrm{m}$. With these assumptions the scaling units of length and frequency take the values $a_{0}=\lambda /[4 \pi \sin (\theta / 2)]=2.52 \mu \mathrm{m}$ and $\omega_{0}=2 \pi \times 224 \mathrm{~Hz}$. A stable bright soliton typically created in the experiment [14] contains $\mathcal{N} \approx 5 \times 10^{3}$ atoms, which corresponds to $N \approx 2.62$ in our units. We note that by changing the angle $\theta$, it is possible to achieve smaller or larger values of $a_{0}$, and hence of $N$, for the same value of $\mathcal{N}$.

At $t=0$, i.e. in the absence of the lattice, the shape of the soliton is given by the exact solution of the GP equation,

$$
\Psi\left(x, x_{0}, 0\right)=(N / 2) \operatorname{sech}\left[(N / 2)\left(x-x_{0}\right)\right],
$$

where $x_{0}$ is the initial position of the soliton, and the motion of the soliton is free. If we assume that the addition of a weak ratchet potential does not affect the soliton shape during its evolution, then the Hamiltonian description of the mean field allows us to treat the matter-wave soliton as an effective classical particle [16] that moves in the effective potential,

$$
\begin{gathered}
V_{\mathrm{eff}}\left(x_{0}, t\right)=\frac{1}{N} \int_{-\infty}^{\infty}\left|\Psi\left(x, x_{0}, 0\right)\right|^{2} V(x, t) d x= \\
=\frac{\pi}{N} V_{0} f(t)\left[\frac{\cos x_{0}}{\sinh (\pi / N)}+2 \frac{\cos \left(2 x_{0}+\phi\right)}{\sinh (2 \pi / N)}\right] .
\end{gathered}
$$

Using Eq.(4) and the equation of motion for the soliton position, $d^{2} x_{0} / d t^{2}=-d V_{\text {eff }} / d x_{0}$, the soliton velocity can be obtained straightforwardly. The instantaneous shape of the effective potential, shown in Fig. 1 for $f(t)=1$, becomes closer to the shape of the optical lattice, $V(x, t)$ (Fig. 1. thin solid line), as $N$ grows and the soliton becomes more localized. For small $N$ the second term in (44) becomes exponentially smaller than the first one, and hence the effective potential becomes practically symmetric, as demonstrated by a solid thick line in Fig. 1.

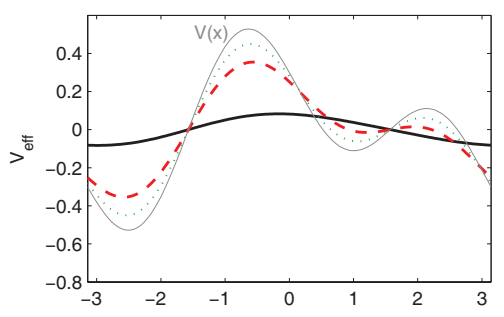

FIG. 1: (Color online) Effective potential $V_{\text {eff }}$ vs. soliton position $x_{0}$, for $N=1$ (solid line), $N=3$ (dashed) and $N=5$ (dotted). Thin solid line is the optical lattice potential $V(x, t)$. Parameters are: $V_{0}=0.3, \phi=\pi / 2, f(t)=1$

The effective-particle approximation (EPA) predicts that the cumulative velocity of the collective excitation, $\bar{v}=(1 / T) \int_{0}^{T} v(t) d t$, is a function of the number of atoms contained in the soliton $(N)$. As can be seen in Fig. 2, this prediction is in good agreement with the numerical results obtained from the GP model, where we have used $T \approx 10^{3} \times 2 \pi / \omega$. As Fig. 2 demonstrates, for a fixed initial position of the soliton, $x_{0}$, there is a sharp transition between a regime where a soliton oscillates between neighboring wells but is not transported [Fig. 2(b)], and a regime where the soliton acquires a ballistic motion [Fig. 2(c)]. The simulations of ballistic motion are performed with the periodic boundary conditions, however we make sure that the tails of the soliton do not overlap. The EPA model also predicts that the velocity of the ballistic motion tends to a constant value as $N \rightarrow \infty$ (and $T \rightarrow \infty$ ), which is qualitatively confirmed by numerical simulations.

The dependence of the cumulative velocity $\bar{v}$ on the number of atoms is also a function of the driving frequency, $\omega$ [see inset in Fig. (2)]. For high frequencies the velocity vanishes as $v \propto \omega^{-1}$, as the soliton becomes less and less affected by the rapidly oscillating potential. For the moderate driving frequencies used here the soliton does not radiate, and small variations of its shape do not 
lead to particle loss. In the limit of small $\omega$ the soliton strongly interacts with the lattice and may break up. In the effective-particle model the trajectory of the soliton in this case is chaotic and a detailed study of its behavior is beyond the scope of this paper.
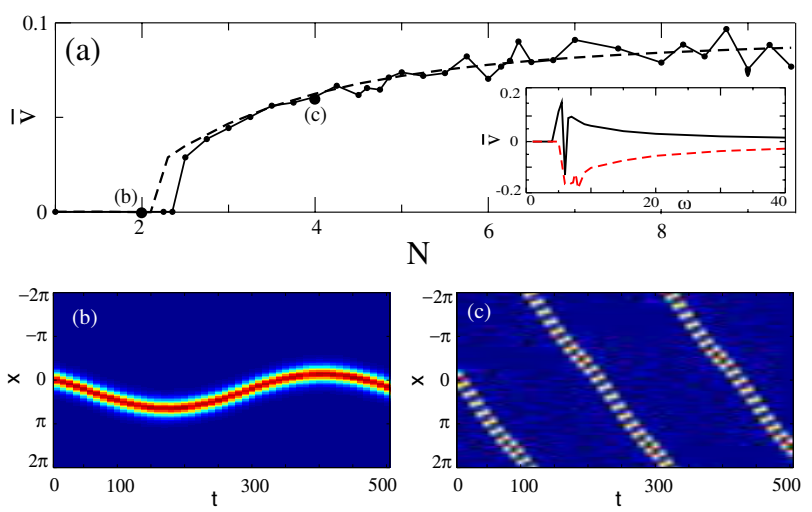

FIG. 2: (Color online) (a) Cumulative velocity $\bar{v}$ vs. number of atoms in the soliton, $N$, calculated using Eq.(1) (solid line) and EPA (dashed); $\bar{v}=1$ corresponds to $3.5 \mathrm{~mm} / \mathrm{s}$. Parameters are: $V=0.3, \phi=\pi / 2, \omega=10, x_{0}=0$. Inset: Cumulative velocity $\bar{v}$ vs. driving frequency $\omega$, for $N=4$ and $x_{0}=0$ (solid line) and $x_{0}=-\pi / 2$ (dashed line). (b,c) Density plot of the mean field evolution, $|\psi(x, t)|^{2}$, shown for the corresponding points at $N=2$ and $N=4$ in (a).

The dependence of the soliton velocity on atom number is a general feature that occurs for symmetric periodic potentials as well, however the precise relationship between velocity and atom number depends on the initial position of the soliton relative to the lattice. Since the space inversion symmetry of the lattice is broken, we can expect that averaging over all initial soliton positions, $x_{0}$, will lead to a directed soliton current. To demonstrate this effect, in Fig. 3 we plot the soliton velocity averaged over all initial positions, $\langle\bar{v}\rangle=(1 / 2 \pi) \int_{0}^{2 \pi} \bar{v} d x_{0}$, as a function of the number of atoms. Although the average velocity is always non-zero, one can identify two different regimes of the ratchet dynamics depending on the number of atoms, as discussed below.

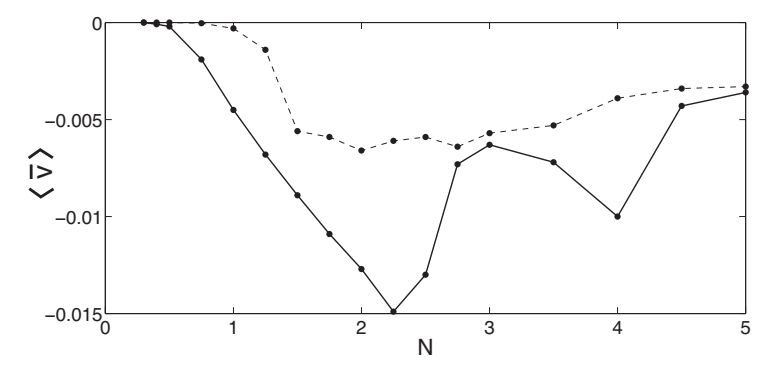

FIG. 3: (a) Average velocity $\langle\bar{v}\rangle$ vs. number of atoms in the soliton, $N$, calculated using the GP model (solid line) and EPA (dashed). Parameters are: $V_{0}=0.3, \phi=\pi / 2, \omega=10$.
As seen in Fig. 3, for small values of $N$ the EPA results and numerical soluton of Eq.(1) disagree both on the onset of the ratchet effect and on its magnitude. For $N<2.5$ the soliton's size is comparable to or larger than a period of the optical lattice. Hence it is more accurately described as a wavepacket than an effective particle. The details of the soliton response to the flashing potential are best seen by examining the dependence of the cumulative velocity $\bar{v}$ on the initial position of the center-of-mass, $x_{0}$. In Figs. $4(\mathrm{a}, \mathrm{b})$ we show this response for $N=1$ and $N=2$. Interestingly the numerical results show that the soliton has either no cumulative velocity, or moves in only one direction. As a result, for $N<2.5$ a soliton attains a much larger average velocity than that predicted by the EPA theory (see Fig. 3). In fact the EPA incorrectly predicts that the soliton can move in both directions depending on its initial position. We also note that for $N=1$ the cumulative velocity expected from the EPA is almost symmetric around $x_{0}=0$ as expected in the limit of small $N$ due to the symmetry of the effective potential (see Fig 1).
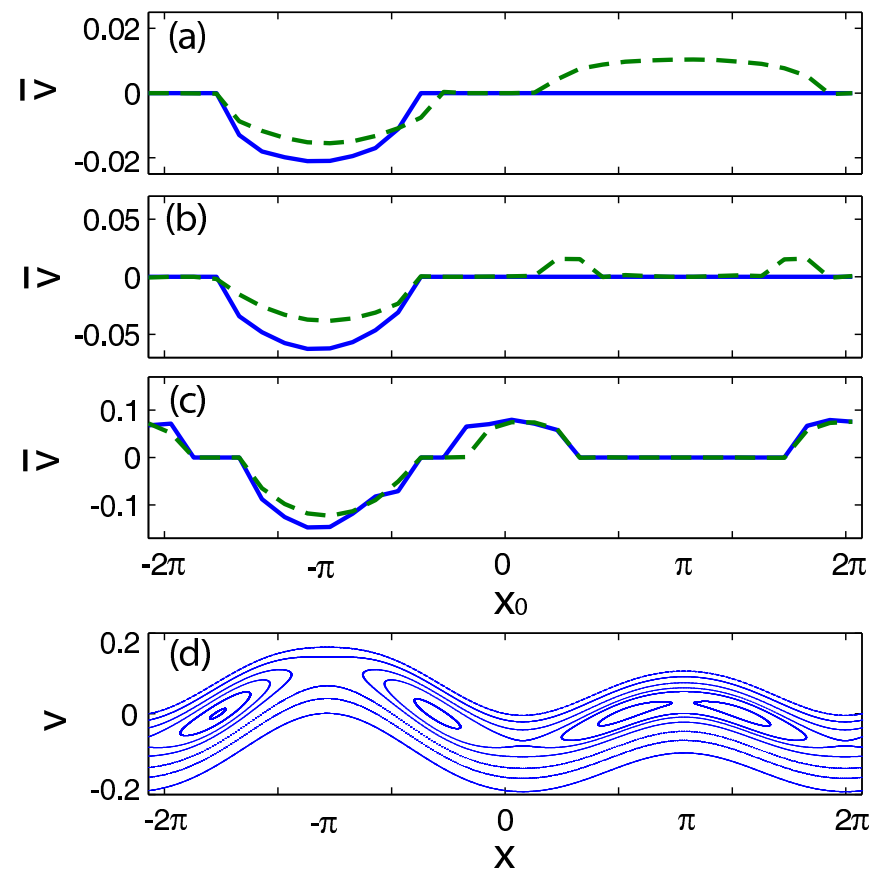

FIG. 4: (Color online) (a) Cumulative velocity, $\bar{v}$, vs. soliton initial position, $x_{0}$, for (a) $N=1$; (b) $N=2$; (c) $N=5$; calculated from the numerical solution of the GP equation (solid line) and EPA (dashed). (d) Poincaré section for the effective-particle model in (c). Parameters are: $V_{0}=0.3$, $\phi=\pi / 2, \omega=10$.

For large values of $N$, corresponding to a strongly localized matter-wave soliton, the ratchet dynamics is well described by the EPA. In Fig. 3 we observe a good agreement between the ratchet velocity predicted by the effective particle model and that found from solving Eq.(1) numerically. Similarly, in Fig. 4(c) we see a good agree- 
ment between the numerics and the EPA in the details of the cumulative velocity dependence on $x_{0}$. In Fig. 4(d) we show the Poincaré section corresponding to the effective particle results of Fig. 4(c). Two different types of trajectories are observed, transporting or nontransporting. A comparison between Figs. 4(c and d) shows a clear correlation between cumulative velocity in Fig. 4(c) and either non-transporting trajectories (correlating with the zero-velocity) or transporting trajectories (correlating with either positive or negative velocity).

As shown above, solitons with different $N$ travel at different ratchet-induced speeds even if their relative initial position in the lattice is the same. Multiple soliton collisions could therefore be realized if the ratchet potential was combined with a toroidal trap [17]. As can be seen in Fig. 5(a) a larger moving soliton can then induce the transport of a smaller soliton which would otherwise not be transported by the ratchet. This is due to the fact that each collision incrementally changes the soliton's position in the phase space and its original motion can thus be dramatically modified if it is eventually moved from a non-transporting to a transporting trajectory. The driving has little effect on the actual near-elastic soliton collisions because of its small amplitude and fast variation.

If the solitons have equal values of $N$, collisions can occur only if they have different relative positions in the lattice, and therefore different speeds. In this case however, the interaction between the solitons is strong due to the large interaction energy. Each collision therefore induces a much more pronounced transition to a different phase-space trajectory. This is evident in Fig. 5(b) where we can see dramatic changes in the soliton velocities after collisions. In this scenario, the spatial shift that solitons acquire during each collision may lead to an effective averaging over $x_{0}$ after multiple collisions. As a result, a nonzero total average current can, in principle, be observed for a sufficiently large number of collisions or for a sufficiently large number of interacting solitons.

In conclusion, we have studied the ratchet dynamics of bright solitons in Bose-Einstein condensates and demonstrated that solitons may either be transported through the potential or oscillate, depending on their initial position relative to the lattice. For small atom numbers, the soliton transport occurs in one direction only, while larger solitons may be transported in either direction. We show that the rate of transport for a given initial position is atom-number dependent, with solitons that contains more atoms typically moving faster. Importantly, we find that averaging over all soliton initial positions reveals an overall directed soliton current, and show a good qualitative agreement between the numerical results and an effective particle approximation. Finally, we illustrate how the ratchet potential affects interactions between solitons containing the same or different number of atoms. Collisions can cause an instantaneous transition between different trajectories in the phase space,
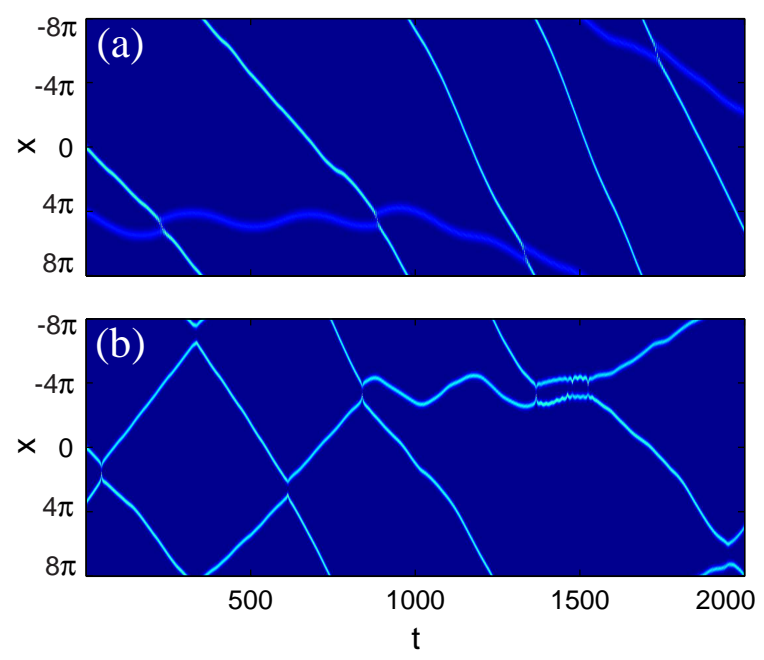

FIG. 5: (Color online) (a) Collision between two soliton, one with $N=4$ with initial position $x_{0}=0$ and the other one with $N=2.2$ and initial position $x_{0}=4 \pi$. (b) Collision between two soliton with $N=4$ located at $x_{0}=0$ and $x_{0}=3 \pi+1.2$. Parameters are: $V_{0}=0.3 . \phi=\pi / 2, \omega=10$.

including that between non-transporting and transporting trajectories. This effect could potentially be used for directed transport or spatial filtering of solitons based on the atom number. This work is supported by the Australian Research Council (ARC).

[1] R.D. Astumian and P. Hänggi, Phys. Today 55, No. 11, 33 (2002).

[2] P. Reimann, Phys. Rep. 361, 57 (2002).

[3] P. Reimann et al., Phys. Rev. Lett. 79, 10 (1997); J. Lehmann et al., ibid. 88, 228305 (2002); G.G. Carlo et al., Phys. Rev. Lett. 94, 164101 (2005).

[4] H. Schanz et al., Phys. Rev. Lett. 87, 070601 (2001); T.S. Monteiro et al., ibid. 89, 194102 (2002); S. Denisov et al., cond-mat/0607558 (2006).

[5] S. Flach et al., Phys. Rev. Lett. 84, 2358 (2000).

[6] R. Gommers et al., Phys. Rev. Lett. 96, 240604 (2006).

[7] D. Poletti et al. Phys. Rev. A 76, 023421 (2007).

[8] O. Romero-Isart and J.J. Garcia-Ripoll, 0709.3463v1.

[9] L. Morales-Molina and S. Flach, arXiv:0710.1339 (2007).

[10] M. Sadgrove et al., Phys. Rev. Lett. 99, 043002 (2007).

[11] I. Dana et al., Phys. Rev. Lett. 100, 024103 (2008).

[12] M. Salerno, N. Quintero, Phys. Rev. E 65, 025602 (2002).

[13] A. V. Gorbach et al., Opt. Lett. 31, 1702 (2006); H. Sakaguchi, B.A. Malomed, Physica D 183, 282 (2003).

[14] L. Khaykovich et al., Science 296, 1290 (2002).

[15] T. Salger et al. Phys. Rev. Lett. 99, 190405 (2007).

[16] R. Scharf and A.R. Bishop, Phys. Rev. E 47, 1375 (1993).

[17] K. Helmerson et al., Nucl. Phys. A 790, 705c (2007). 